\title{
Dermal Filler Injections Mimic Tumor Activity during Immune Checkpoint Inhibition
}

\section{Zulfiya Syunyaeva Kathrin Kahnert Diego Kauffmann-Guerrero Rudolf Maria Huber Amanda Tufman}

Division of Respiratory Medicine and Thoracic Oncology, Department of Internal Medicine V, University of Munich (LMU), Thoracic Oncology Centre Munich, Munich, Germany; Comprehensive Pneumology Center, Member of the German Center for Lung Research (DZL), Munich, Germany

A 53-year-old female patient with advanced nonsmall-cell lung cancer presented with cutaneous nodules and hardened skin folds of the neck (Fig. 1a) during treatment with nivolumab. A PET-CT scan after 5 nivolumab infusions revealed a partial tumor response; however, new FDG uptake was seen along the musculi sternoclei- domastoidei (Fig. 1b, c). The patient showed no clinical signs of autoimmune thyroiditis and TSH, T3, and T4 were within normal range.

The patient reported several cosmetic procedures including dermal filler injections to the anterior neck to reduce visible wrinkles and folds. The dermal filler consist-
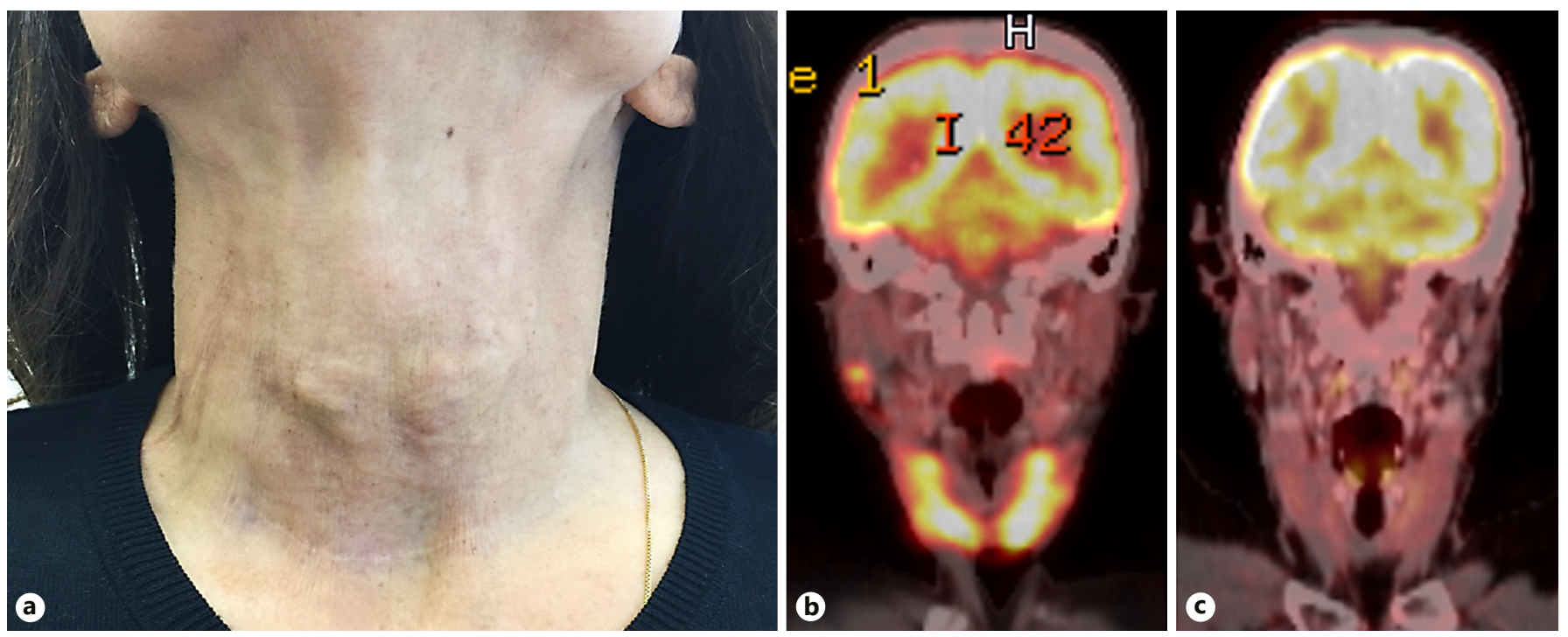

Fig. 1. a Cutaneous nodules and hardened skin folds of the neck. FDG uptake along the musculi sternocleidomastoidei under nivolumab (b) and prior to nivolumab (c). 
ing of carboxymethyl cellulose and polycaprolactone microspheres (Ellansé ${ }^{\circledR}$ ) [1] was injected 15 months before initiation of immunotherapy and was well tolerated.

Autoimmune colitis developed during nivolumab and was treated with systemic glucocorticoids. This resulted in resolution of the gastrointestinal symptoms as well as improvement of the skin nodules. We interpret the subcutaneous FDG uptake as a nivolumab-related foreign body reaction to the dermal filler. Foreign body reactions following dermal filler can be triggered by systemic infections, drugs or immunotherapy [2-4]. One case report describes a foreign body reaction during ipilimumab for malignant melanoma [2], but to date there are no reports of comparable reactions during nivolumab.
Since immunotherapy is now broadly used in cancer treatment and side effects are diverse, physicians should be aware of possible foreign body reactions. The FDG avidity of these reactions in ${ }^{18} \mathrm{~F}$-FDG PET-CT scans highlights a possible pitfall in treatment monitoring and can occur after different cancer treatments including surgical treatment with the use of oxidized cellulose hemostatic agents for bleeding control [5]. Physicians interpreting scans should be aware of patients' previous cosmetic procedures, and patients treated with immunotherapy should be informed about this potential side effect.

\section{References}

Dermal Filler Injections Mimic Tumour Activity during Immunotherapy
1 Christen M-O: Ellansé Safety: Sinclair Pharma. https://ellanse.com/wp-content/uploads/ sites/2/2017/04/1605-Ellanse\%CC\%81eSafety-report.pdf.

2 Bisschop C, Bruijn MS, Stenekes MW, Diercks GF, Hospers GA: Foreign body reaction triggered by cytotoxic T lymphocyte-associated protein 4 blockade 25 years after dermal filler injection. Br J Dermatol 2016;175: 1351-1353.

3 De Boulle K, Heydenrych I: Patient factors influencing dermal filler complications: prevention, assessment, and treatment. Clin Cosmet Investig Dermatol 2015;8:205-214.
4 Lemperle G, Gauthier-Hazan N, Wolters M, Eisemann-Klein M, Zimmermann U, Duffy DM: Foreign body granulomas after all injectable dermal fillers: part 1. Possible causes. Plast Reconstr Surg 2009;123:1842-1863.

5 Zhou Q, West DG, Shelley-Fraser G, Medford AR: Foamy macrophage deposition in lymph nodes mimicking lung cancer recurrence diagnosed via endobronchial ultrasound-guided transbronchial needle aspiration. Respiration 2015;90:426-429. 